\title{
Origins and renormalization of the superparticle spectrum
}

\author{
Nir Polonsky ${ }^{\mathrm{a}} * \dagger$ \\ ${ }^{a}$ Department of Physics and Astronomy, Rutgers University, Piscataway, NJ 08855-0849, USA
}

The importance of Yukawa contributions to the renormalization of the spectrum in non-minimal supersymmetric models is illustrated in the cases of explicit lepton number violation (leading to the possibility of singly produced sneutrinos at LEP energies), an intermediate scale singlet neutrino and negative mass squared parameters (possibly modifying fine-tuning considerations), and a grand-unified sector. The relevance of model-dependent renormalization to the supersymmetric flavor problem is emphasized. Sources of the flavor problem, some of which are newly identified, as well as possible solutions, are discussed and classified. It is then shown that gravitational interactions could lead (via a quadratically divergent singlet) to simple realizations of some of the low-energy frameworks that attempt to resolve the flavor problem.

\section{Report No. RU-97-48}

\section{Introduction}

Low-energy supersymmery offers an attractive, consistent, and well motivated extension of the Standard Model (SM). Whether supersymmetry is indeed realized at the weak scale will be determined by experiment during the next decade or so. In particular, the characterization of the spectrum of the superpartners of the ordinary fermions (sfermions) and of the gauge and Higgs bosons (gauginos and Higgsinos) has to await their discovery. (Some hopes that precision measurements would give clear indirect indications of certain light sparticles, e.g., from $Z \rightarrow b \bar{b}$, proved premature [1][2].)

Once the various masses and mixing angles are measured, their correlations would allow one to disentangle and determine the spectrum parameters and to learn about its high-energy origins. The latter task is complicated by the sensitivity of the spectrum evolution from high to low energies to new interactions and to new sectors in the theory (which couple to the ordinary particles). Such sensitivities, on the other hand, offer unique opportunities to discover new interactions

\footnotetext{
*Work supported by NSF grant No. PHY-94-23002.

$\dagger$ Talk presented at the Fifth International conference on Supersymmetries in Physics, Philadelphia, May 1997.
}

and sectors.

In the absence of solid experimental evidence we are left, at present, with the following missions: (i) Surveying and understanding the available parameter space and the possible spectrum patterns. (ii) Employing theoretical criteria in order to identify the more interesting and motivated possibilities. The optimal search strategies that are and will be employed are highly dependent on the specific spectrum pattern one is searching for. They are particularly dependent on the identity of the lightest (and the next to lightest) supersymmetric particle ((N)LSP) and on the mass hierarchy, which determine the collider signature (missing energy, hard photons, hadronic activity, etc.) and the various decay modes, respectively. Hence, the identification and classification of the possible and the more motivated patterns is crucial for a fruitful experimental search and analysis (if indeed supersymmetry is realized in nature). Furthermore, creating a map between different spectra and the different scenarios of supersymmetry breaking which they parameterize, may enable us to have an indirect glimpse at the supersymmetry breaking (SSB) sector and at any other coupled sectors in the foreseeable future.

The issues we alluded to above are vast and 
are intensively (but far from completely) studied. Here, we would like to comment on both the possible origins and the renormalization of the spectrum. In particular, we will stress that neither the model dependent spectrum renormalization (or evolution) nor the "spectrum pattern - SSB sector" map are unique. We will also comment on the supersymmetric flavor problem and on its role as a selection criterion for models.

We note in passing that one could also choose to study the dimensionless couplings in the Lagrangian whose form is dictated by supersymmetry. Any small deviations from the supersymmetric identities (e.g., between the gauge and gaugino couplings to matter and between the gauge and quartic couplings) could also shed light on heavy and extended sectors. These issues and the related superoblique parameters were discussed in these proceedings [3] and in recent publications [ $[5]$, and will not be explored here.

\section{Minimal supergravity: A reference framework}

Before discussing any complexities, it is instructive to review the simplistic but phenomenologically consistent and highly predictive framework of minimal supergravity. The minimal supergravity framework will also serve us as a convenient reference point. (See Ref. [6] for review and references to the many works that have established this framework in the last two decades.)

The minimal supergravity framework specifies all three functions that define the effective $N=1$ supersymmetric theory: $W, K$, and $f_{\alpha \beta}$. The superpotential $W=W_{\text {hidden }} \oplus W_{\text {observable }}$ is a direct sum of SSB sector superpotential $W_{\text {hidden }}$ and of the superpotential corresponding to the (twoHiggs doublet) SM (suppressing flavor indices and using self explanatory notation), $W_{\text {observable }}=$ $h_{E} H_{1} L E^{c}+h_{D} H_{1} Q D^{c}-h_{U} H_{2} Q U^{c}-\mu H_{1} H_{2}$. (Note that the limit [7] $\mu \rightarrow 0$ was recently excluded by searching for an excess of $W^{+} W^{-}$-like events in the LEP2 $\sqrt{s}=161 \mathrm{GeV}$ data [8].)

The most simple example of the hidden SSB sector superpotential is that of Polonyi [9], $W_{\text {hidden }}=m^{2}(Z+\beta)$ where $m^{2} \sim \mathcal{O}\left(m_{\text {weak }} M_{P}\right)$ determines the scale of supersymmetry breaking and $\beta$ is a dimensionful constant of the order of the Planck mass $M_{P}$. ( $\beta$ is tuned to cancel the cosmological constant). More generally, one requires $\langle Z\rangle \sim M_{P}, W_{\text {hidden }} \sim m_{\text {weak }} M_{P}^{2}$, and $(\partial W / \partial Z) \sim m_{\text {weak }} M_{P}$. The gravitino mass $m_{3 / 2} \sim W_{\text {hidden }} / M_{P}^{2} \sim M_{S U S Y}^{2} / M_{P}$ is $m_{3 / 2}=$ $\mathcal{O}\left(m_{\text {weak }}\right)$ in this case. ( $M_{S U S Y}$ is the scale of supersymmetry breaking in the hidden sector and $M_{S U S Y}=m$ in the Polonyi model.)

The Kahler function $K=\Lambda_{a b} \Phi^{a} \Phi^{b \dagger}$ is minimal with $\Lambda_{a b}=\delta_{a b}$ and does not mix the hidden and observable sectors. The above assumptions regarding $W$ and $K$ are sufficient to ensure that SSB is mediated to the observable sector gravitationally (and softly) and that the (tree-level) boundary conditions for the scalar potential parameters are universal (and proportional), i.e., at Planckian scales $V_{\mathrm{SSB}}=m_{0}^{2} \sum_{i}\left|\phi_{i}\right|^{2}+\left(B \mu H_{1} H_{2}+\right.$ $A_{0} h_{i j k} \phi^{i} \phi^{j} \phi^{k}+$ h.c.) with the soft SSB parameters $m_{0} \sim A \sim B \sim m_{3 / 2} \sim m_{\text {weak }}$. We also used $\Lambda_{a b}=\delta_{a b}$ in the summation (otherwise the first term is not universal). In addition, if assuming gauge coupling unification (or universal gauge kinetic functions $f_{\alpha \beta}^{i}=\delta_{\alpha \beta} / g^{2}\left[1+\mathcal{O}\left(M_{P}^{-1}\right)\right]$ at Planckian scales) then the gauginos also have a common mass at the high-energy boundary which is parameterized by $M_{1 / 2} \sim m_{3 / 2}$. (It is generated, in principle, from the field-dependent terms in $f_{\alpha \beta}$.) Various mechanisms allow one to also realize $\mu=\mathcal{O}\left(m_{3 / 2}\right)$, so that all dimensionful parameters in the potential of the observable sector are of the order of the weak scale.

Neglecting Yukawa interactions, the renormalized scalar spectrum simply reads $m_{i}^{2}(Q)=m_{0}^{2}+$ $a_{i}(Q) M_{1 / 2}^{2}$, where the momentum-dependent positive-definite coefficients $a_{i}(Q)$ are charge dependent but flavor blind. Also, the trilinear terms in the scalar potential are diagonalized simultaneously with the SM Yukawa matrices. Both results lead to suppression of new contributions to flavor changing neutral currents (FCNC). Such contributions could be unacceptably large for an arbitrary spectrum (the supersymmetric flavor problem) and their successful suppression is crucial for the validity of any model.

The missing ingredient which is required in order to reproduce the Standard Model (SM) La- 
grangian properly is the negative squared mass in the Higgs potential. Indeed, the $m_{H_{2}}^{2}$ parameter is differentiated from all other squared mass parameters once we include the Yukawa interactions. For simplicity, and without loss of generality, let us assume that only the $t$-quark Yukawa coupling $h_{t}$ is relevant. (More generally, the $b$ quark and $\tau$-lepton couplings may not be negligible.) Then, the well-known one-loop evolution of $m_{H_{2}}^{2}$ (and of the coupled parameters $m_{U_{3}}^{2}$ and $m_{Q_{3}}^{2}$ ) with respect to the logarithm of the momentum is given by

$\frac{\partial m_{H_{2}}^{2}}{\partial \ln Q}=\frac{1}{8 \pi^{2}}\left(3 h_{t}^{2} \Sigma_{m^{2}}-3 g_{2}^{2} M_{2}^{2}-g_{1}^{2} M_{1}^{2}\right)$,

and

$\frac{\partial m_{U_{3}}^{2}}{\partial \ln Q}=\frac{1}{8 \pi^{2}}\left(2 h_{t}^{2} \Sigma_{m^{2}}-\frac{16}{3} g_{3}^{2} M_{3}^{2}-\frac{16}{9} g_{1}^{2} M_{1}^{2}\right)$,

$$
\begin{array}{r}
\frac{\partial m_{Q_{3}}^{2}}{\partial \ln Q}=\frac{1}{8 \pi^{2}}\left(h_{t}^{2} \Sigma_{m^{2}}-\frac{16}{3} g_{3}^{2} M_{3}^{2}-3 g_{2}^{2} M_{2}^{2}\right. \\
\left.-\frac{1}{9} g_{1}^{2} M_{1}^{2}\right),
\end{array}
$$

where $\Sigma_{m^{2}}=\left[m_{H_{2}}^{2}+m_{Q_{3}}^{2}+m_{U_{3}}^{2}+A_{t}^{2}\right]$, and we denote the $\mathrm{SM} \mathrm{SU}(3), \mathrm{SU}(2)$ and $\mathrm{U}(1)$ gauge coupling and gaugino mass by $g_{3,2,1}$ and $M_{3,2,1}$, respectively. Given the heavy $t$-quark one has $h_{t} \sim 1 \sim g_{3}$. (In fact, typically $h_{t}>g_{3}$ at high energies.) While QCD loops still dominate the evolution of the stop squared masses $m_{Q_{3}}^{2}$ and $m_{U_{3}}^{2}$, Yukawa loops dominate the evolution of $m_{H_{2}}^{2}$. On the one hand, the stop squared masses and $\Sigma_{m^{2}}$ increase with the decreasing scale. On the other hand, the greater they increase the more the Higgs squared mass decreases with scale, and it is rendered negative at the weak scale. The $B \mu$ Higgs doublet mixing ensures that both Higgs doublets have non-vanishing expectation values. This is a simplistic description of the well known mechanism of radiative electroweak symmetry breaking. In fact, the sizeable $h_{t}$ typically renders the Higgs squared mass too negative and some (fine?) tuning (usually of $\mu$ ) is required in order to extract the precisely known electroweak scale correctly.
Before considering extended and more complicated scenarios, let us recall the main features of the model:

- The only interactions between the SSB and observable sectors are gravitational, i.e., the SSB sector is hidden. (It is simple, minimal, attractive, but not unique.)

- Large Yukawa couplings and Yukawa loops generate a negative mass squared. (It is a desirable dynamical mechanism, it predicts that the $t$-quark is sufficiently heavy, and it is very attractive.)

- One obtains scalar mass universality at the Planckian boundary at the price of strong assumptions regarding the Kahler potential (a desirable result but unattractive assumptions).

Below, we will examine similar effects of (strong) Yukawa interactions due to new interactions and new sectors and their consequences, look at the issue of universality and the corresponding flavor problem, and point out new mechanisms which were recently proposed for gravitational mediation of SSB [10] which are significantly different from the supergravity framework described above.

\section{Imprints of Yukawa interactions on the scalar spectrum}

Similar dynamics to those leading to the successful radiative electroweak symmetry breaking appear in many other cases. The imprints of such dynamics on the scalar potential and spectrum depend on the strength of the couplings, the corresponding quantum numbers, and the relevant energy regime. Once we have understood the importance of loops $\propto h_{t}^{2}$, it is straightforward to observe and understand the imprints of extended Yukawa interactions. We will discuss three examples of such extensions.

\section{New Interactions}

It is well known that $W_{\text {observable }}$ could be extended by either lepton or baryon number violating Yukawa terms, which are allowed by all gauge 
symmetries, $\lambda L L E^{c}+\lambda^{\prime} L Q D^{c}$ and $\lambda^{\prime \prime} U^{c} D^{c} D^{c}$, respectively [11]. Phenomenological constraints allow for some of the couplings to be $\mathcal{O}(1)$. If such couplings exist then, e.g.,

$$
\frac{\partial m_{L}^{2}}{\partial \ln Q}=\frac{1}{8 \pi^{2}}\left(3 \lambda^{\prime 2} \Sigma_{m^{2}}^{L}-3 g_{2}^{2} M_{2}^{2}-g_{1}^{2} M_{1}^{2}\right),
$$

where $\Sigma_{m^{2}}^{L}=\left[m_{L}^{2}+m_{Q}^{2}+m_{U}^{2}+A_{\lambda^{\prime}}^{2}\right]$. Eq. (何) is obvious from eq. (11), and all other relevant equations are modified in a similar fashion. Hence, the slepton doublet tends to be light (for a large coupling) due to wavefunction renormalization $\propto \lambda^{\prime 2} m_{\text {squark }}^{2}$. The successful radiative symmetry breaking mechanism implies that if $\lambda^{\prime} \sim$ $\mathcal{O}\left(h_{t}\right)$ then the relevant slepton doublet is light (most probably $L_{\tau}$, which we will also denote as $L_{3}$ ). (Note that the $\lambda^{\prime}$ and the ordinary Yukawa couplings could have simultaneous quasifixed points [12].) These observations are independent of any supergravity assumptions.

One finds in this case a highly interesting situation: A strongly interacting light slepton doublet. Together with Erler and Feng we recently explored such a scenario in some detail [13]. Specifically, we considered the operators $\lambda_{131} L_{1} L_{3} E_{1}^{c}+\lambda_{333}^{\prime} L_{3} Q_{3} D_{3}^{c}$. One has the constraints $\lambda_{131}<0.1\left[m_{\text {slepton }} / 100 \mathrm{GeV}\right]$ (from charged current universality) [14], $\lambda_{333}^{\prime}<$ $0.96\left[m_{\text {squark }} / 300 \mathrm{GeV}\right]$ (from $B \rightarrow \tau \bar{\nu} X$ ) 13 , and $\lambda_{131} \lambda_{333}^{\prime}<0.075\left[m_{\text {slepton }} / 100 \mathrm{GeV}\right]^{2}$ (from $B \rightarrow$ $e \bar{\nu})$ [13. The constraints allow for a large $\lambda^{\prime}$, consequently leading to the possibility of light (lefthanded) stau $\tilde{\tau}_{L}$ and sneutrino $\tilde{\nu}_{\tau}$. Their masses are related by $\mathrm{SU}(2)$ invariance but are slightly split by the electroweak $D$-terms $m_{\tilde{\tau}_{L}}^{2}-m_{\tilde{\nu}_{\tau}}^{2} \sim$ $-0.77 M_{Z}^{2} \cos 2 \beta>0$ (here $\tan \beta=\left\langle H_{2}\right\rangle /\left\langle H_{1}\right\rangle$ ). Hence, the sneutrino may very well be the LSP (NLSP if the gravitino is the LSP) and $\tilde{\tau}_{L}$ the NLSP, which we will assume in this example.

It is interesting to note that the CP even and odd components of the sneutrino are also slightly split in mass in this case [13]. This is due to a $b$-quark loop $\propto \lambda_{333}^{\prime 2} m_{b}^{2}$ which contributes to the $\tilde{\nu} \tilde{\nu}$ mass term but not to the $\tilde{\nu} \tilde{\nu}^{*}$ term. (The analogue $b-\tilde{b}$ loop generates a neutrino mass, but

\footnotetext{
${ }^{3}$ We implicitly assume here the basis in which the sneutrino interaction with the down-type quarks is flavor diagonal. See Ref. 13 for a general discussion.
}

it depends also on left-right mixing parameters.) The split effect was found to be negligible in our case. (For other examples, see Ref. [15].)

The large coupling and the light $\tilde{\tau}_{L}$ allow for $t \rightarrow b \tilde{\tau}_{L} \rightarrow b b c$. The corresponding branching ratio (and hence, mass and coupling) can be constrained most efficiently by the reconstruction of $M_{W}$ in lepton + jets double $b$-tagged top events at the Tevatron (from the untagged jets). The analysis, however, is currently constrained by the limited statistics. Turning to the sneutrino, $e^{+} e^{-} \rightarrow \tilde{\nu}_{\tau} \rightarrow b b$, a fit to electroweak data assuming a light sneutrino can improve the SM fit. It suggests $m_{\tilde{\nu}_{\tau}}=91.79 \pm 0.54 \mathrm{GeV}$ with $\lambda_{131}=\mathcal{O}(0.01)$ and $\lambda_{333}^{\prime}=\mathcal{O}(0.5)$ (from the favorable contributions to the $b$ branching ratio $R_{b}$ and forward-backward asymmetry $\left.A_{F B}^{0}(b)\right)$. These results are highly non-trivial and should be compared to the typically poor fits in the lepton number conserving cases [2]. Of course, this does not provide any evidence for $m_{\tilde{\nu}_{\tau}} \simeq M_{Z}$, but only indicates a window which is slightly preferred by current data. More relevant are the search opportunities at LEP2. The singly produced sneutrino may have a wide resonance $\sim 6 \mathrm{GeV} \times \lambda_{333}^{\prime 2}\left[m_{\tilde{\nu}_{\tau}} / 100 \mathrm{GeV}\right]$. When the effect of radiative returns (i.e., initial state hard photon radiation) to the sneutrino threshold is also included, one finds that a low-luminosity scan with only a few steps can either discover the sneutrino at LEP2 energies (up to the kinematic limit) or significantly improve the constraints on the couplings. In certain regions of the parameter space the anticipated $\sqrt{s} \sim 180-190 \mathrm{GeV}$ runs may suffice for discovery of the sneutrino. (For detailed examples and discussion, see Ref. [13].)

It should be noted that the sneutrino tail would enable one to explore sneutrinos slightly above the kinematic limit. Also, the $e^{+} e^{-} \rightarrow \tilde{\nu}_{\tau} \rightarrow$ $b b$ cross section is approximately $\propto \lambda_{131}^{2} / \lambda_{333}^{\prime 2}$. Therefore, even if one rescales down both couplings (within a reasonable range), our results are only weakly affected. It emphasizes the possible role of LEP if hints of an excess of neutral current events at HERA are proved to be correct and are interpreted within a supersymmetric framework [16]. (One may have to generalize the analysis to include $b d$ and $b s$ production.) 


\section{New fields}

Extensions of $W_{\text {observable }}$ by new SM singlet and vector-like fields at the weak or at intermediate scales are also widely considered. A singlet superfield could couple (via a Yukawa term) to $H_{1} H_{2}$ or to $L_{2}$, while new exotic charged fields could mix with the ordinary SM fields. Their couplings could again affect the spectrum evolution. (Exotic sectors could also contribute to the superoblique parameters of Cheng et al. [1].) Additional weak-scale $U(1)^{\prime}$ models, which could be motivated by string theory, often imply such new states and were discussed in Ref. 17]. Here, however, we will discuss only the case of an intermediate-scale right-handed (singlet) neutrino $N: W_{\text {observable }} \rightarrow W_{\text {observable }}+$ $M_{N} N^{2}-h_{N} H_{2} L N$.

As an example we have chosen the possible interplay between the SSB parameters $m_{N}^{2}$ and $m_{H_{2}}^{2}$. While the SSB contribution to the intermediate-scale singlet sneutrino mass is negligible, the size and sign of $m_{N}^{2}$ could be important for the evolution of the Higgs SSB parameters. This is the case if $h_{N}=\mathcal{O}(1)$, as is suggested by grand-unified models which typically predict $h_{N_{\tau}}=h_{t}$ at the grand-unification scale. In our example, we will further assume $m_{N}^{2}<0$ and some (non-minimal) supergravity scenario (and $\left.\left|m_{N}^{2}\right| \sim \mathcal{O}\left(m_{3 / 2}^{2}\right)\right)$.

It was already shown in Ref. [7] that the evolution of $m_{H_{2}}^{2}$ can be controlled and moderated by carefully choosing $m_{Q_{3}}^{2}, m_{U_{3}}^{2}<0$ at the Planckian boundary. The physical stop squared masses are still positive due to gluino loops (which are summed by the effective potential or the renormalization), and $m_{t}^{2} F$-terms. One replaces the tuning of electroweak parameters (e.g., $\mu$, as discussed above) with tuning of boundary conditions. This can be understood intuitively: By allowing negative squared masses at high scales, the $\Sigma_{m^{2}}$ terms in eqs. (1) - (3) can be dialed to be small, zero (which is in some sense a quasifixed point in the absence of gaugino loops), or even negative. Due to the gaugino loops, $\Sigma_{m^{2}}$ can change sign in the course of integration.

Such dialing can be done more easily in the singlet neutrino framework [7]. Here, $m_{N}^{2}<0$ could be a boundary condition or could be achieved in the course of renormalization between the Planckian and intermediate scales,

$\frac{\partial m_{N}^{2}}{\partial \ln Q}=\frac{1}{8 \pi^{2}}\left(2 h_{N}^{2} \Sigma_{m^{2}}^{N}\right)$,

where $\Sigma_{m^{2}}^{N}=\left[m_{H_{2}}^{2}+m_{L}^{2}+m_{N}^{2}+A_{N}^{2}\right]$. As noted above, $\Sigma_{m^{2}}$-type terms oscillate in sign and hence the squared mass parameters are bounded from below. For simplicity, let us assume that $m_{N}^{2}<0$ is a boundary condition. In either case, its effect on the physical heavy sneutrino mass is negligible. We note in passing that if we did not introduce the $M_{N}$ supersymmetric mass term, a radiatively generated $m_{N}^{2}<0$ would lead to $\langle N\rangle \sim m_{3 / 2}$ and to new possibilities of neutrino mass generation 18.

The Higgs mass evolution is now given by

$$
\begin{array}{r}
\frac{\partial m_{H_{2}}^{2}}{\partial \ln Q}=\frac{1}{8 \pi^{2}}\left(3 h_{t}^{2} \Sigma_{m^{2}}+h_{N}^{2} \Sigma_{m^{2}}^{N}\right. \\
\left.-3 g_{2}^{2} M_{2}^{2}-g_{1}^{2} M_{1}^{2}\right) .
\end{array}
$$

Clearly, by carefully choosing the boundary condition for $m_{N}^{2}$ (even for $m_{Q_{3}}^{2}, m_{U_{3}}^{2}>0$ ) one can control and moderate $m_{H_{2}}^{2}$ renormalization at some initial momentum interval, affecting the required tuning of electroweak Higgs potential parameters. Indirectly, $m_{N}^{2}<0$ would also lead to lighter stops (via eqs. (2) and (3i)). We conclude that the sneutrino presence is reflected in this example in the magnitude of the Higgs potential parameters. Of course, $m_{L}^{2}$ renormalization is also affected. However, we will return to this point after the following and last example.

\section{New sectors}

We briefly mentioned above the possibility of new electroweak and intermediate scale sectors. Here, however, we would like to recall some of the consequences of a grand-unified (GUT) sector with strong Yukawa interactions in supergravity models 19 21. We will assume in this example supergravity and that the supergravity scale (i.e., the integration boundary) is above the GUT scale $M_{G}$.

One can distinguish two leading effects: Those which are due to large SM Yukawa couplings, and 
in particular effects related to quark-lepton unification, and those which are due to new large Yukawa couplings which couple heavy states to light states. The former is manifested in, e.g.,

$\frac{\partial m_{10_{3}}^{2}}{\partial \ln Q}\left(Q>M_{G}\right)=\frac{1}{8 \pi^{2}}\left(3 h_{t}^{2} \Sigma_{m^{2}}^{10}-\ldots\right)$,

where we use $\mathrm{SU}(5)$ classification, $10_{3} \quad \ni$ $\left(Q_{3}, U_{3}, E_{3}\right), \Sigma_{m^{2}}^{10}=\left[m_{H_{2}}^{2}+2 m_{10}^{2}+A_{t}^{2}\right]$, and we omitted the gauge terms. (In some extended models $L_{3}$ is also unified with the $t$-quarks and " $m_{L_{3}}^{2}\left(Q>M_{G}\right)$ " is also renormalized by $h_{t}^{2}$ terms.) The effects are enhanced by the diverging $h_{t}\left(M_{G}\right) \sim 2$. Eq. (7) is valid between the GUT and supergravity (= string?) scales. The latter effects are due to operators of the form $\lambda_{\Phi} \Phi H_{1} H_{2}$ where $\Phi$ is a heavy $\mathcal{O}\left(M_{G}\right)$ field. In minimal models at least one field $\langle\Phi\rangle \sim M_{G}$ and $\lambda_{\Phi} \sim 1$ are required in order to render the color triplet GUTpartners of the Higgs doublets sufficiently heavy (so that radiative proton decay is sufficiently suppressed). If indeed $\lambda_{\Phi} \sim 1$ then

$$
\begin{aligned}
\frac{\partial m_{H_{2}}^{2}}{\partial \ln Q}\left(Q>M_{G}\right)= & \frac{1}{8 \pi^{2}}\left(C^{\Phi} \lambda_{\Phi}^{2} \Sigma_{m^{2}}^{\Phi}\right. \\
& \left.+3 h_{t} \Sigma_{m^{2}}^{10}-\ldots\right),
\end{aligned}
$$

where $\Sigma_{m^{2}}^{\Phi}=\left[m_{H_{2}}^{2}+m_{H_{1}}^{2}+m_{\Phi}^{2}+A_{\Phi}^{2}\right]$ and $C^{\Phi}$ is a group theory factor (similarly for $m_{H_{1}}^{2}$ ). Due to the large group dimensions and representations, all effects are enhanced by large group-theory factors. The combination of large couplings and large group theory factors can more than compensate for the short integration interval.

It is interesting to note that the effects are somewhat complimentary: $\lambda_{\Phi} \ll 1$ would suppress the second effect but it leads to thresholds (corresponding to the GUT-partners of the Higgs doublets) below the unification scale. In this case there are threshold corrections $\propto h_{t}^{2}$ to the third family sfermion SSB mass parameters (particularly to the slepton ones) which enhance the $h_{t}$ effects [22]. The threshold corrections are independent of the assumption of renormalization beyond the GUT scale, which may not hold, for example, in non-perturbative string theories.

It was recently realized that the heavy $t$-quark can lead to dramatic effects in the renormaliza- tion of the third family sfermion and Higgs boson SSB parameters between the GUT and supergravity scales (in particular, when combined with the effects of large $\lambda_{\Phi}$ couplings). Even if one introduces the minimal supergravity universal boundary conditions at the supergravity scale, they are wildly violated at the GUT scale due to their evolution eqs. (7) and (8) [21]. In Ref. [21] it was emphasized that the Higgs potential parameters (and hence radiative symmetry breaking and fine-tuning considerations) as well as typical spectrum correlations are significantly modified. The latter may enable one to probe such scenarios. (See also Ref. [23].) It is interesting to note that even if the $t$-quark were not heavy, successful radiative symmetry breaking could be achieved in this and in the previous singlet neutrino example due to $\lambda_{\Phi} \Sigma_{m^{2}}^{\Phi}\left(h_{N} \Sigma_{m^{2}}^{N}\right)$ contributions to the evolution.

Ref. 24] focused on the corrections $\propto h_{t}^{2}$ to $m_{E_{3}}^{2}$, which introduce flavor dependent corrections to the slepton spectrum. It was found that the corresponding contributions of slepton loops to leptonic FCNC processes, particularly $\mu \rightarrow e \gamma$, may not be negligible. As we alluded to above, such corrections also arise in the case of the singlet neutrino. Unlike the example here, the $h_{N}^{2}$ corrections split the left-handed slepton spectrum according to the hierarchies of the singlet neutrino couplings and SSB parameters. (For a recent analysis, see Ref. [25]). Potential contributions to flavor violation, including $\mu \rightarrow e \gamma$ [26], also constrain the couplings $\lambda$ and $\lambda^{\prime}$ of our first example (see above). The couplings $\lambda^{\prime}$ also splits the left-handed slepton spectrum, leading to a new class of contributions to FCNC in lepton number violating models. These contributions are not directly proportional to the $\lambda^{\prime 2}$, are similar to the ones discussed in Ref. [24,25], and have not been studied yet.

\section{The Flavor Problem}

We concluded the previous section with the discussion of sparticle loop contributions to leptonic FCNC processes. The contributions discussed above were due to radiatively generated non- 
universalities in the slepton spectrum. Moreover, fermion and sfermion mass matrices are not diagonalized simultaneously for generic nonuniversal (and non-proportional) boundary conditions for the soft SSB parameters $m_{i}^{2}$ (and $A_{i j k}$ ), leading to flavor non-diagonal fermionsfermion-gaugino (and sfermion-sfermion-Higgs) couplings. In these cases sparticle loops can contribute to strongly constrained FCNC processes, in particular, those involving the first and second family fermions.

The unwanted contributions to FCNC processes can be suppressed in two obvious ways: (a) Arranging for simultaneous diagonalization of fermion and sfermion mass matrices; or $(b)$ suppression of dangerous loops in the case of arbitrary sfermion masses by raising the relevant sparticle mass scale (e.g., $B R(\mu \rightarrow e \gamma) \propto$ $\left.1 / m_{\text {slepton }}^{4}\right)$. In fact, these are the two mechanisms that are also invoked to suppress dangerous contributions in the lepton number violating models discussed above. However, there one can also suppress the new and arbitrary Yukawa couplings $\lambda$ and $\lambda^{\prime}$, while here the ordinary Yukawa couplings are determined by the fermion spectrum and are not arbitrary. These solutions are perhaps the most important theoretical criteria in constructing models for SSB mediation to the observable sector. (See our discussion above of minimal supergravity.) We will propose in the following section new gravitational frameworks that can achieve these goals 10 at the minimal price of a low-energy singlet (rather than strong assumption on the form of $K$ ). Before doing so, we will briefly review some of the solutions, their advantages, and their drawbacks.

\footnotetext{
$\overline{4}$ Non-universalities $\propto h_{t}^{2} \ln \left(M_{P} / m_{\text {weak }}\right)$ in the squark spectrum can be typically ignored due to small 13 and 23 CKM mixing and hadronic uncertainties. This is not the case here, given the extremely sensitive search for, e.g., $\mu \rightarrow e \gamma$.

${ }^{5}$ The previous examples implicitly assume non-diagonal lepton Yukawa matrices, as is suggested by grandunification. Any explicit predictions, e.g., for $B R(\mu \rightarrow e \gamma)$ (as well as for radiative proton decay $p \rightarrow K l$ ) must assume specific Yukawa textures.
}

\section{Simultaneous diagonalization}

Two classes of sfermion mass matrices which commute with the Yukawa matrices are often discussed - universal mass matrices (which are proportional to the identity) and aligned mass matrices (sfermion mass matrices which are proportional to the Yukawa matrices). Here, we will discuss only the former mechanism.

Imposing universality in the general supergravity framework leads to the minimal framework described above. It requires strong assumptions on the structure of the Kahler potential, and in particular, suppression of any light-heavy mixing which generically leads to $\Lambda_{a b} \neq \delta_{a b}$ [27]. It was recently pointed out that even if such assumptions are made, gravitational interactions typically generate radiatively flavor non-diagonal masses $\left(\Delta m^{2} / m^{2}\right) \sim\left(N / 16 \pi^{2}\right)$, unless the model has a stringy $T$-duality [10]. (Here $N=\mathcal{O}(10)$ counts the number of massless states.) These radiative corrections are gravitational and are different from the model-dependent field-theory radiative corrections that we discussed in previous examples and which are still in effect. The dilaton limit in perturbative string theory corresponds to a (calculable) example of a universal model [28]. It is also subject to (stringy) radiative corrections [29]. In addition, no convincing realization of this limit in string theory exists. More generically, perturbative string theory predicts a nonuniversal spectrum which depends on the modular weights of the fields. (This seems also to be the situation in non-perturbative string theories [30].) In the a priori unlikely case of universal weights one would recover the supergravity limit $\Lambda_{a b}=\delta_{a b}$.

It is sufficient, however, to require only charged-dependent flavor universality. In (any) supergravity framework this is the case if $M_{1 / 2} \gg$ $m_{i}(0)$ where $m_{i}(0)$ is a generic boundary condition for the soft SSB scalar masses. In string theory this would be the case if there is a symmetry that relates the gauge charges and the modular weights [10]. This is also the situation in the framework of low-energy supersymmetry breaking if SSB is mediated to a messenger sector (i.e., $\left.W \neq W_{\text {hidden }} \oplus W_{\text {observable }}\right)$ and then to the observable sector via gauge interactions (i.e., gauge 
mediation of SSB). (For reviews and references, see Ref. 31,32].) In fact, only the nature of the second mediation is relevant for the issue of universality, a point which is relevant to the discussion in the next section. In all such examples one expects mass hierarchy according to charges, e.g., heavy squarks and significantly lighter sleptons.

Some elaboration on the the low-energy messenger/gauge-mediation framework, which offers an alternative phenomenological framework to supergravity models, is in place. The gaugino masses result in this framework from the messenger (SM) gauge interactions and are generated at one-loop, $m_{\text {gaugino }}(\Lambda) \sim\left(g^{2} / 16 \pi^{2}\right) \Lambda$. The scalar squared masses, on the other hand, are generated only at two-loops, $m_{i}^{2}(\Lambda) \sim\left[\left(g^{2} / 16 \pi^{2}\right) \Lambda\right]^{2}$ (since one typically forbids direct interactions between the messengers and the ordinary matter fields which could be flavor dependent). Therefore, gaugino masses and scalar masses are of the same order of magnitude without any special assumptions on the gauge kinetic functions $f_{\alpha \beta}^{i}$. (This is also the case in the dilaton limit [28] discussed above and, apparently, in non-perturbative string theory 30.) The scale $\Lambda$ typically corresponds to the SSB mediation scale, which is roughly of the order of the SSB scale. One finds that such models are phenomenologically valid if $\Lambda \sim 10^{5}$ $\mathrm{GeV}$, which is well below the unification and intermediate scales. (The heavy squarks compensate in this case for the shorter integration interval in the radiative symmetry breaking mechanism.) On the other hand, in the low energy SSB case, $m_{3 / 2} \sim W_{\text {hidden }} / M_{P}^{2} \sim \Lambda^{3} / M_{P}^{2} \rightarrow 0$. As a result, supergravity mechanisms to generate $\mu \sim m_{3 / 2}$ are irrelevant (like most other supergravity contributions). Radiative generation of $\mu$ at low energy typically implies the generation of " $B \mu$ " at the same loop order and " $B \mu$ " $/ \mu \sim \Lambda$. Such a situation leads to an unacceptable finetuning. This is perhaps the most severe phenomenological difficulty in this framework 32. We will return to the gauge mediation/messenger framework in the next section.

\section{Arbitrary sfermion mass matrices}

The phenomenological " $2-1$ " framework that underlies these ideas is straightforward and was reviewed in Ref. [33]. One distinguishes a heavy decoupled sector which contains the first and second family sfermions ("2" generations) and a lighter sector which contains the Higgs fields, as well as the gauginos and third family ("1" generation) fields which couple to the Higgs fields (see eq. (II)). The most dangerous sparticle contributions to FCNC processes (and to $\mathrm{CP}$ odd observables) decouple, while a priori no extreme fine-tuning is required in the Higgs potential. The realization of such a framework, however, is not as straightforward. Such models could be realized in the framework of dynamical low-energy supersymmetry breaking [33] or by embedding an anomalous $\mathrm{U}(1)$ horizontal symmetry in a supergravity framework 34. A new realization of this framework will be discussed in the next section.

The two sectors, of course, are not truly decoupled. Terms which couple their renormalization and are typically suppressed by small hypercharge and Yukawa couplings, two-loop factors and by the universality assumption, are now enhanced by the heavy masses and their arbitrariness. Hence, in practice there is a trade off between fine-tuning and an upper bound on the decoupled sector scale (and hence, the spectrum arbitrariness) [35]. (The bound depends on the specific realization and on the relevant integration interval.) It appears that more severe constraints on the decoupling scale are due to negative physical stop squared masses [36]. However, note that in the derivation of the latter constraints, Yukawa terms were omitted even though negative squared-mass parameters appear (either radiatively or as boundary conditions). Therefore, such omission is not justified [37]. (See an example in the previous section).

We note in passing that this and some of the previous frameworks contain heavy states that may be probed by the superoblique parameters. The implications of the flavor problem and its solutions to the dimensionless couplings and to the superoblique parameters are discussed in Ref. [4].

\section{Gravitational triggering models}

Solutions to the flavor problem, which we discussed above, often assume contributions to the 
scalar spectrum which are independent of the Kahler potential, so that no strong assumptions on the form of $K$ need to be made. (But see our proposal of a symmetry relating modular weights and gauge charges in string theory. In general, some symmetry assumptions have to be made somewhere.) The gauge mediation (messenger) mechanism and some realizations of the decoupling mechanism further assume that gravitational interactions have no significant role in the mediation of SSB. That is, it is usually assumed that gravitational interactions are negligible for the determination of the spectrum if supersymmetry is broken at low-energies. This intuitive assumption holds in general, but it fails in the particular case of a light global and local (universal) singlet.

Here, we would like to show in a simple example how such a singlet can lead to gravitational triggering of a gauge mediation framework. We will apply the general framework to two specific examples: An ordinary messenger framework and a horizontal messenger framework. Our discussion follows the work of Ref. [10]. (Ref. [10] also contains an application which we do not discuss here and which attempts to solve the "low-energy $\mu$ problem" described in the previous section.)

The observable sector is extended to include an universal singlet, $S=s+\theta^{2} F_{s}$, and a vector-like pair of non-singlet fields $V$ and $\bar{V}$. The specific toy model that we will consider here is given by the superpotential

$$
W_{\text {observable }} \rightarrow W_{\text {observable }}+\frac{\kappa}{3} S^{3}+\lambda_{S} S V \bar{V} .
$$

The Kahler potential is given by (suppressing higher orders in $M_{P}^{-1}$ )

$$
K=\sum_{I}\left(1+\alpha_{I} \frac{S+S^{\dagger}}{M_{P}}\right) \Phi_{I} \Phi_{I}^{\dagger}
$$

Obviously, we assume no $\mathcal{O}\left(M_{P}\right)$ dimensionful parameters for the singlet field, a situation which can be understood in the context of a string theory or duality transformations. The $D$-terms

$$
\int d^{2} \theta d^{2} \bar{\theta} e^{K / M_{P}^{2}} K
$$

lead to a quadratically divergent (tadpole) contribution to the scalar potential [38],

$\Delta V_{\mathrm{SSB}}=-\frac{M_{S U S Y}^{4}}{M_{P}} s+h . c .$,

where we implicitly assume no Planckian values for suspersymmetry breaking fields. (The general situation, as well as our phase choice and the ambiguity due to dimensionless couplings and loop factors, are discussed in Ref. [10].) Including the usual $F$-terms $|\partial W / \partial S|^{2}=\kappa^{2} s^{4}$ (which is the only relevant term if $2 \lambda_{S}>\kappa$ ) and neglecting any other supergravity terms aside from the tadpole, we have

$s=\left(\frac{M_{S U S Y}^{4}}{4 \kappa^{2} M_{P}}\right)^{\frac{1}{3}}, F_{s}=\kappa s^{2}$,

and the scalar messenger squared-mass matrix

$M_{v \bar{v}}^{2} \sim\left(\begin{array}{cc}\lambda_{S}^{2} s^{2} & \lambda_{S} F_{s} \\ \lambda_{S} F_{s}^{*} & \lambda_{S}^{2} s^{2}\end{array}\right)$.

The diagonal term is a supersymmetric mass term, i.e., the corresponding fermions have a similar Dirac mass term $\lambda_{S} s \tilde{v} \tilde{\bar{v}}$. Similarly, field dependent masses are induced for $s$ and for its fermion partner $\tilde{s}$, and are given by replacing $\lambda_{S}$ with $\kappa / 3(\kappa)$ in the diagonal (off-diagonal) terms in eq. (14).

The fields $S$ and $V, \bar{V}$ are suitable to play the role of the singlet and non-singlet messenger fields in a gauge mediation framework with (the usual [31]) $\Lambda=F_{s} / s \sim s$. Note the minimality of this framework as SSB is transmitted to the messenger fields gravitationally, i.e., $W=W_{\text {hidden }} \oplus W_{\text {observable }}$. Only the fields $S, V, \bar{V}$ are needed and the messenger sector is absorbed in the observable sector. The quadratic divergences allow for the gravitational mediation of (low-energy) SSB to the messenger singlet, hence triggering the usual gauge-mediation framework.

\section{A messenger model}

By examination of the gravitationally triggered gauge mediation framework (13) - (14), we find that $M_{S U S Y} \sim 10^{8} \mathrm{GeV}$ corresponds to $\Lambda \sim 10^{5} \mathrm{GeV} .\left(M_{S U S Y} \sim 10^{5} \mathrm{GeV}\right.$, had we allowed Planckian values for supersymmetry breaking fields.) Hence, we find the usual messenger 
model framework 31, 32. It depends only on the scale of SSB, not on its details.

\section{A horizontal messenger model}

If $M_{S U S Y} \sim 10^{10} \mathrm{GeV}$, supergravity masses are not negligible. However, from eq. (13) we have in this case $\Lambda \sim 10^{7} \mathrm{GeV}$, which leads to $\mathcal{O}(10$ $\mathrm{TeV})$ masses in the corresponding gauge mediation framework. (Corrections $\sim m_{3 / 2}$ to (13) are still negligible.) This numerology hints at a new realization of decoupled " $2-1$ " models. We can assume that $V$ and $\bar{V}$ are SM singlets but are charged under some horizontal symmetry that distinguishes the first two families (which transform non-trivially) from the third family and Higgs fields (which transform trivially). In this case, the former would acquire $\mathcal{O}(10 \mathrm{TeV})$ masses via (horizontal) messenger gauge loops while the latter would have only $\mathcal{O}\left(m_{\text {weak }}\right)$ supergravity masses. (In the case of Planckian values for supersymmetry breaking fields, $\Lambda \sim M_{S U S Y} \sim 10^{10}$ $\mathrm{GeV}$ can be offset by an extremely weak horizontal gauge coupling.)

\section{Summary}

In summary, we discussed some issues in the evolution and sources of the superparticle spectrum. The flavor problem was shown to be affected by the former and to be a useful criterion in classifying and selecting the latter. Various unrelated effects, from light sneutrinos to radiative contributions to FCNC, have been shown to originate from similar mechanisms. New sources of flavor violation were identified. The flavor problem (which is often attributed to the ad hoc arbitrariness of the Kahler potential) was discussed in some detail, and it was shown that some of its solutions can be triggered gravitationally (from the divergent Kahler potential interactions of a universal singlet).

Underlying our discussion and the various examples are the potential difficulties in the theoretical interpretations of future measurements. Indeed, each interpretation requires one to specify a framework for the renormalization of the spectrum (1.e., the non-hidden sector) and a framework to the mediation of SSB to the observable sector (i.e., the boundary and the parameterization of the boundary conditions).

In the previous section we considered examples of gravitationally triggered frameworks which suggest that the supergravity, $M_{S U S Y} \sim$ $10^{10-11} \mathrm{GeV}$, and the low-energy SSB framework, $M_{S U S Y} \sim 10^{5-7} \mathrm{GeV}$, only mark the most simple (and perhaps most attractive) limits on a scale chart. (See Ref. 39] for different examples.) In the simplest versions of these limits the spectrum is described by a small number of parameters, $\left(m_{0}, A_{0}, B, M_{1 / 2}, \mu\right)$ and $(\Lambda, B, \mu)$, respectively. The horizontal messenger example suggests a linear combination of (new) gauge mediated and (non-minimal) supergravity contributions to the spectrum, and some states beyond the reach of future colliders. The parameterization of the spectrum may not be as simple in this case, and some of the parameters may never be characterized by experiment (but see Ref. 㕶). Even if the simple low energy SSB + gauge mediation framework describes the data in a satisfactory fashion, our messenger example suggests that its interpretation is not unique.

In addition, the need to specify a renormalization framework (one usually assumes the minimal supersymmetric SM framework) can lead to new difficulties. On the other hand, it provides a rare opportunity to learn about the nonhidden sectors at intermediate and high energies. Hence, our window on the observable/non-hidden sector comes at the price of a potentially less "clear view" of the SSB/hidden sector. The correct renormalization framework may reveal itself in the identity of the (N)LSP, as well as in the enhancement of rare processes such as $\mu \rightarrow e \gamma$ (though in both examples the observations may not have a unique interpretation).

It is important to keep in mind that analysis within the most simple frameworks, though it is a well motivated first step, is far from unique and it may partially or completely fail. Such failure may be due to simplistic assumptions, and alternative interpretations should then be pursued. Particularly, we argued that the LEP collaborations should exploit their physics potential and search for a singly produced sneutrino if conventional sparticle searches fail. 
The examples explored in this contribution are based on old and new works in various collaborations with H.-C. Cheng, J. Erler, J. L. Feng, C. Kolda, H. P. Nilles, A. Pomarol, and S. Thomas.

\section{REFERENCES}

1. The status of supersymmetric models is reviewed in these proceedings by $\mathrm{H}$. E. Haber.

2. D.Pierce, in these proceedings.

3. J. L. Feng, in these proceedings; L. Randall, E. Katz, and $\mathrm{S}$. Su, in these proceedings.

4. H.-C. Cheng, J. L. Feng, and N. Polonsky, hep-ph/9706438.

5. H.-C. Cheng, J. L. Feng, and N. Polonsky, hep-ph/9706476; M. M. Nojiri, D. M. Pierce, and Y. Yamada, hep-ph/9707244.

6. P. Nath, in these proceedings.

7. J. L. Feng, N. Polonsky, and S. Thomas, Phys. Lett. B 370 (1996) 95.

8. M. Gruwe, talk presented at the OPAL collaboration workshop on Higgs boson and supersymmetry searches, The Weizmann Institute, Rehovot, Israel, May 14-19, 1997.

9. J. Polonyi, Budapest Report KFKI-93 (1977).

10. H. P. Nilles and N. Polonsky, hep-ph/9707249.

11. For a recent review see, for example, G. Bhattacharyya, in Supersymmetry '96, Nucl. Phys. Proc. Supp. 52A (1997) 83.

12. V. Barger, M. S. Berger, R. J. N. Phillips, and T. Whormann, Phys. Rev. D 53 (1996) 6407.

13. J. Erler, J. L. Feng, and N. Polonsky, Phys. Rev. Lett. 78 (1997) 3063.

14. V. Barger, G. Giudice, and T. Han, Phys. Rev. D (1989) 2987.

15. M. Hirsch, H. V. Klapdor-Kleingrothaus, and S. G. Kovalenko, Phys. Lett. B 398 (1997) 311; Y. Grossman and H. E. Haber, Phys. Rev. Lett. 78 (1997) 3438.

16. For a review, see G. Altarelli, in these proceedings.

17. J. R. Espinosa, in these proceedings.

18. N. Polonsky, work in progress.

19. P. Moxhay and K. Yamamoto, Nucl. Phys. B256 (1985) 130.

20. L. J. Hall, V. A. Kostelecky, and S. Raby,
Nucl. Phys. B267 (1986) 415.

21. N. Polonsky and A. Pomarol, Phys. Rev. Lett. 73 (1994) 2292; ibid. Phys. Rev. D 51 (1995) 6532.

22. N. Polonsky, Phys. Rev. D 54 (1996) 4537.

23. R. Arnowitt and P. Nath, hep-ph/9701325.

24. R. Barbieri and L. J. Hall, Phys. Lett. B 338 (1994) 212.

25. J. Hisano, T. Moroi, K. Tobe, and M. Yamaguchi, Phys. Rev. D 53 (1996) 2442.

26. For example, see M. Chaichian and K. Huitu, Phys. Lett. B 384 (1996) 157.

27. S. K. Soni and H. A. Weldon, Phys. Lett. 126B (1983) 215.

28. V. S. Kaplunovsky and J. Louis, Phys. Lett. B 306 (1993) 269.

29. J. Louis and Y. Nir, Nucl. Phys. B447 (1995) 18.

30. I. Antoniadis, in these proceedings; E. Dudas, in these proceedings; H. P. Nilles, M. Olechowski, and M. Yamaguchi, hep-th/9707143.

31. C. Kolda, in these proceedings.

32. M. Dine, in these proceedings.

33. A. Nelson, in these proceedings.

34. G. Dvali and A. Pomarol, Phys. Rev. Lett. 77 (1996) 3728.

35. S. Dimopoulos and G. F. Giudice, Phys. Lett. B 357 (1995) 573.

36. N. Arkani-Hamed and H. Murayama, hepph/9703259.

37. C. Kolda and N. Polonsky, work in progress.

38. For a complete reference list see Ref. [10]. Here, we will use some of the results of J. Bagger, E. Poppitz, and L. Randall, Nucl. Phys. B455 (1995) 59.

39. Other models were considered recently by, e.g., K. Choi, Phys. Rev. D 54 (1996) 6591; R. N. Mohapatra and S. Nandi, Phys. Rev. Lett. 79 (1997) 181; S. Raby, hep-ph/9702299. See also contributions by E. Poppitz and P. Trivedi, and by H. Murayama. 